\title{
Fast Root-finding of Nonlinear Equations in Geometric Computation
}

\author{
Changchun Geng, Zhong Li, Tianhe Zhou, Bin Yang \\ Department of Mathematical Sciences \\ Zhejiang Sci-Tech University \\ Hangzhou, 310018, China \\ e-mail: lizhong@zstu.edu.cn
}

\begin{abstract}
Computing the roots of polynomials is an important issue in various geometric problems. In this paper, we introduce a new family of iterative methods with sixth and seventh order convergence for nonlinear equations (or polynomials). The new method is obtained by combining a different fourth-order iterative method with Newton's method and using the approximation based on the divided difference to replace the derivative. It can improve the order of convergence and reduce the required number of functional evaluations per step. Numerical comparisons demonstrate the performance of the presented methods.
\end{abstract}

Keywords - Newton's method; Convergence order; Divided difference; Non-linear equation

\section{INTRODUCTION}

Efficient and robust algorithms for finding the solutions of (systems of) polynomial equations are frequently used in geometric computing, modeling and processing, such as curves and surface intersection, surface rendering by ray-tracing, collision detection, etc [1].

Recently, Barton and Juttler provided the quadratic clipping to compute all the roots of a univariate polynomial equation within an interval whose convergence order is 3 for single roots [2]. Liu et al generalized it to the cubic clipping to compute the roots of polynomial equations which can attain the fourth-order convergence for single roots [3]. Both methods are based on the degree reduction of the polynomial approximation and may suffer numerical instability. They actually can be converted into the problem for computing roots of non-linear equations. In this paper, we study the iterative method to find a simple root $\alpha$ to get $f$ $(\alpha)=0$ and $f^{\prime}(\alpha) \neq 0$ of a non-linear equation $f(x)=0$.

Classical Newton's iteration is the well-known method which converges quadratically for a single non-linear equation. Some higher order iterative methods have recently been proposed and analyzed for solving nonlinear equations. In order to improve the order of convergence, a known technique consists of the combining two iterative methods of orders $p$ and $q$, to obtain a method of order $p q$ [4], respectively. However, the process increases the number of functional evaluations per step. We can use some approximations of the derivatives to reduce the number of functional evaluations, still maintaining the convergence order as high as possible to improve the efficiency of the proposed method.

For example, Parhi and Gupta [5] composed Weerakoon and Fernando's third order method [6] with Newton's method and approximated the derivative by linear interpolation to obtain a sixth-order method. Sharma and Guha [7] derived a one-parameter family of sixth order methods based on Ostrowski fourth order multipoint method. Mir and Zaman in [8] composed Ostrowski's method with Halley's method, introducing a parameter that allowed them to obtain families of iterative methods with sixth, seventh and eighth orders of convergence. Cordero and Hueso [9] provided an iterative method with sixth and seventh order convergence helped by using Kou et al's fourth-order method [10].

In this paper, we present two new three-step iterative methods for solving nonlinear equations with sixth and seventh convergence order. The new methods are obtained by composing known methods with Newton's method and using an adequate approximation for the last derivative. The efficiency index of these methods are 1.565 and 1.627 , which makes them competitive and the new methods need only three functions and one first derivative, respectively.

\section{DESCRIPTION OF THE METHODS}

In order to compose new methods, we recall some notions about divided differences. Let us consider the nonlinear equation $f(x)$ which is a real function defined on $R$, where $R$ is the smallest interval containing $n+1$ distinct nodes $\left[x_{0}, x_{1}, \ldots, x_{n}\right]$. The divided difference $f\left[x_{0}, x_{1}, \ldots, x_{n}\right]$ with $n$ th-order is defined as follows:

$$
\begin{aligned}
& f\left[x_{0}\right]=f\left(x_{0}\right), \\
& f\left[x_{0}, x_{1}\right]=\frac{f\left[x_{1}\right]-f\left[x_{0}\right]}{x_{1}-x_{0}}, \\
& \quad \ldots \\
& f\left[x_{0}, x_{1}, \cdots, x_{n}\right]=\frac{f\left[x_{1}, x_{2}, \cdots, x_{n}\right]-f\left[x_{0}, x_{1}, \cdots, x_{n-1}\right]}{x_{1}-x_{0}} .
\end{aligned}
$$

When $f^{n}(x)$ is continuous in the smallest interval $R$ which contains $x_{0}, x_{1}, \ldots, x_{n}$, we have 


$$
\begin{array}{r}
f\left[x_{0}, x_{1}, \cdots, x_{n}\right]=\int_{0}^{1} d s_{1} \int_{0}^{s_{1}} d s_{2} \cdots \int_{0}^{s_{n-1}} f^{n}\left(\left(1-s_{1}\right) x_{0}\right. \\
\left.+\left(s_{1}-s_{2}\right) x_{1}+\cdots+\left(s_{n-1}-s_{n}\right) x_{n-1}+s_{n} x_{n}\right) d s_{n} .
\end{array}
$$

In our previous work, we obtained the following iterative method

$$
\left\{\begin{array}{l}
y_{n}=x_{n}-\frac{f\left(x_{n}\right)}{f^{\prime}\left(x_{n}\right)}, \\
z_{n}=y_{n}-h\left(\mu_{n}\right) \cdot \frac{f\left(y_{n}\right)}{f\left[y_{n}, x_{n}\right]},
\end{array}\right.
$$

where $\mu_{n}=\frac{f\left(y_{n}\right)}{f\left(x_{n}\right)}$ and $h\left(\mu_{n}\right)$ represents a real-valued function.

We have proved that when $h(0)=1, h^{\prime}(0) \neq 1$ and $\mid h^{\prime \prime}$ $(0) \mid<\infty$, the convergence order of the iterative method is three, when $h(0)=1, h^{\prime}(0)=1$ and $\left|h^{\prime \prime}(0)\right|<\infty$, the convergence order of the iterative method is four.

Here, we use the divided difference with Newton's method and (1) to get a new three-step family of iterative methods.

$$
\left\{\begin{array}{l}
y_{n}=x_{n}-\frac{f\left(x_{n}\right)}{f^{\prime}\left(x_{n}\right)}, \\
z_{n}=y_{n}-h\left(\mu_{n}\right) \frac{f\left(y_{n}\right)}{f\left[y_{n}, x_{n}\right]}, \\
x_{n+1}=z_{n}-\frac{f\left(z_{n}\right)}{f\left[z_{n}, y_{n}\right]+f\left[z_{n}, x_{n}, x_{n}\right]\left(z_{n}-y_{n}\right)} .
\end{array}\right.
$$

We have following theorem for the proposed method (2).

Theorem1. Let $\alpha \in I$ be a simple zero of a sufficiently function $f: I \subseteq R \rightarrow R$ in an open interval $I$. If $x_{0}$ is sufficiently close to $\alpha$, then the method defined by (2) have convergence order at six for $h(0)=1, h^{\prime}(0) \neq 1$ and $\mid h^{\prime \prime}$ $(0) \mid<\infty$. Furthermore, when $h(0)=1, h^{\prime}(0)=1$ and $\left|h^{\prime \prime}(0)\right|<\infty$, the order is seven.

Proof: Let $\alpha$ be a single root of $f(x), e_{n}=x_{n}-\alpha$. By using the Taylor expansion around $x=\alpha$ and taking into account $f$ $(\alpha)=0$, we have

$$
\begin{aligned}
& f\left(x_{n}\right)=f^{\prime}(\alpha)\left[e_{n}+c_{2} e_{n}{ }^{2}+c_{3} e_{n}^{3}+c_{4} e_{n}^{4}\right. \\
& \left.+c_{5} e_{n}{ }^{5}+c_{6} e_{n}{ }^{6}+c_{7} e_{n}{ }^{7}+o\left(e_{n}^{8}\right)\right] \text {, } \\
& f^{\prime}\left(x_{n}\right)=f^{\prime}(\alpha)\left[1+2 c_{2} e_{n}+3 c_{3} e_{n}{ }^{2}+4 c_{4} e_{n}{ }^{3}\right. \\
& \left.+5 c_{5} e_{n}{ }^{4}+6 c_{6} e_{n}^{5}+7 c_{7} e_{n}{ }^{6}+0\left(e_{n}{ }^{7}\right)\right], \\
& \text { where } c_{k}=\frac{f^{(k)}(\alpha)}{k ! f^{\prime}(\alpha)}, k=2,3, \cdots \text {. }
\end{aligned}
$$

From above two formulas, we know

$$
\begin{aligned}
& \frac{f\left(x_{n}\right)}{f^{\prime}\left(x_{n}\right)}=e_{n}-c_{2} e_{n}^{2}+\left(2 c_{2}{ }^{2}-2 c_{3}\right) e_{n}^{3}-\left(4 c_{2}^{3}-\right. \\
& \left.7 c_{2} c_{3}+3 c_{4}\right) e_{n}^{4}+\left(8 c_{2}{ }^{4}-20 c_{2}{ }^{2} c_{3}+6 c_{3}^{2}+10 c_{2} c_{4}\right. \\
& \left.-4 c_{5}\right) e_{n}^{5}-\left(16 c_{2}{ }^{5}-52 c_{2}{ }^{3} c_{3}+28 c_{2}{ }^{2} c_{4}-17 c_{3} c_{4}\right. \\
& \left.+33 c_{3}{ }^{2} c_{2}-13 c_{2} c_{5}+5 c_{6}\right) e_{n}^{6}+\left(22 c_{3} c_{5}-36 c_{2}{ }^{2} c_{5}\right. \\
& -6 c_{7}+16 c_{2} c_{6}-92 c_{2} c_{3} c_{4}+12 c_{4}{ }^{2}+72 c_{4} c_{2}^{3}-18 c_{3}^{3} \\
& \left.+126 c_{2}{ }^{2} c_{3}{ }^{2}-128 c_{2}{ }^{4} c_{3}+32 c_{2}{ }^{6}\right) e_{n}^{7}+o\left(e_{n}^{8}\right) .
\end{aligned}
$$

Then we can get the expansions of $d_{n}, f\left(y_{n}\right), \mu_{n}$, $h\left(\mu_{n}\right), f\left[y_{n}, x_{n}\right], z_{n}, f\left(z_{n}\right)$ and $f\left[z_{n}, y_{n}\right]+f\left[z_{n}\right.$, $\left.x_{n}, x_{n}\right]\left(z_{n}-y_{n}\right)$.

After the careful computation, we obtain $e_{n+1}=c_{2}^{3}(-1+h(0))^{2} e_{n}^{4}-2 c_{2}{ }^{2}(h(0)-1)\left(-3 c_{3} h(0)\right.$ $\left.+3 h(0) c_{2}^{2}-h^{\prime}(0) c_{2}^{2}+2 c_{3}-2 c_{2}^{2}\right) e_{n}^{5}+\frac{1}{h(0)}$

$\left(c_{2}\left(-14 h(0) c_{2}{ }^{2} h^{\prime}(0) c_{3}+16 h(0)^{2} c_{2}{ }^{2} h^{\prime}(0) c_{3}\right.\right.$ $-21 c_{2}{ }^{2} h(0) c_{3}+6 c_{2} h(0) c_{4}+16 h(0) c_{2}{ }^{4} h^{\prime}(0)$

$-h(0) h^{\prime \prime}(0) c_{2}{ }^{4}+60 h(0)^{2} c_{2}{ }^{2} c_{3}-15 h(0)^{2} c_{2} c_{4}$

$-41 h(0)^{3} c_{2}{ }^{2} c_{3}-18 h(0)^{2} c_{2}{ }^{4} h^{\prime}(0)+h(0)^{2} c_{2}{ }^{4} h^{\prime \prime \prime}(0)$

$+9 h(0)^{3} c_{2} c_{4}+h(0) h^{\prime}(0)^{2} c_{2}{ }^{4}-c_{3} h(0)^{4} c_{2}{ }^{2}$

$+17 h(0)^{3} c_{2}{ }^{4}+12 h(0)^{3} c_{3}{ }^{2}+2 h(0)^{4} c_{2}{ }^{4}+10 h(0) c_{2}{ }^{4}$

$\left.+c_{2}{ }^{2} c_{3}-28 h(0)^{2} c_{2}{ }^{4}+4 h(0) c_{3}{ }^{2}-16 h(0)^{2} c_{3}{ }^{2}\right) e_{n}{ }^{6}$

$+\left(32 c_{2}{ }^{3} \mathrm{c}_{4}-18 \mathrm{~h}(0)^{3} \mathrm{c}_{2}{ }^{6}+27 \mathrm{~h}(0)^{3} \mathrm{c}_{2}{ }^{4} \mathrm{c}_{3}+\cdots-20 c_{2}{ }^{6}\right) e_{n}{ }^{7}$ $+o\left(e_{n}^{8}\right)$.

We can easily find when $h(0)=1$ and $h^{\prime}(0) \neq 1$, the coefficients of $e_{n}^{4}, e_{n}^{5}$ in (3) are zero, and the coefficients of $e_{n}{ }^{6}$ are not zero. The convergence order of the iterative method (2) is six.

When $h(0)=1$ and $h^{\prime}(0)=1$, the coefficients of $e_{n}^{4}, e_{n}^{5}$ and $e_{n}{ }^{6}$ in (3) are zero, and the coefficients of $e_{n}^{7}$ are not zero. The convergence order of the iterative method (2) is seven.

Remark1. Per iteration of the present method (2) requires three evaluations of the function and one of its first derivative. The efficiency index is usually used to compare different methods in the literature. We recall that the index [11] is defined as $p^{1 / d}$, where $p$ is the order of convergence and $d$ is the total number of function evaluations per iteration. If we assume that all the evaluations have the same cost as function one, we have that the efficiency 
index of our methods are 1.565 and 1.627 , which makes them competitive.

\section{NUMERICAL RESULTS}

In this section, we do the experiment to test the efficiency of our numerical method. All computations were done by using MATLAB. We present some numerical results for various convergent iterative schemes in Table IIII. We compare with Newton's method (NM), the M3 method provided in [4], the PM method proposed in [2], the MK7 Method defined [6] and our new iteration (GM). In our iteration, we set

$$
h\left(\mu_{n}\right)=\frac{\mu_{n}}{1+\mu_{n}}
$$

We remark that chosen for comparison are only the methods which do not require the computation of second or higher derivatives of the function to carry out iterations. We use following test functions

$$
\begin{aligned}
& \text { (a) } f(x)=x \exp \left(x^{2}\right)-\sin (x)^{2}+3 \cos (x)+5 \\
& \alpha \approx-1.207647827130919 . \\
& \text { (b) } f(x)=\exp \left(x^{2}+7 x-30\right)-3 \\
& \quad \alpha \approx 3.083966303731217 . \\
& \text { (c) } f(x)=10 x \exp \left(-x^{2}\right)-1 ; \\
& \quad \alpha \approx 1.6796306104284499 . \\
& \text { (d) } f(x)=(x-1)^{3}-\sqrt{2} \\
& \quad \alpha \approx 2.122462048309373 . \\
& \text { (e) } f(x)=\sin (x)^{2}-x^{2}+1 \\
& \alpha \approx 1.404491648215341
\end{aligned}
$$

We accept an approximate solution rather than the exact root, depending on the precision $\varepsilon$ of the computer. We use the following stopping criteria: (1). $\left|x_{n+1}-x_{n}\right|<\varepsilon,(2)$. $\left|f\left(x_{n}\right)\right|<\varepsilon$. When the stopping criterion is satisfied, $x_{n+1}$ is taken as the exact root. For numerical illustrations in this section we use the fixed stopping criterion $\varepsilon=10^{-14}$.

In Table I-III, $\mathrm{COC}$ is the computed order of convergence, $\mathrm{N}$ denotes the number of iterations to approximate the root to 14 decimal places, NOFE denotes the number of function evaluations, NA means that the method can not get the root of the equation.

TABLE I. COMPARISON OF THE COMPUTED ORDER OF CONVERGENCE FOR DIFFERENT ITERATIVE METHODS

\begin{tabular}{|c|c|c|c|c|c|c|}
\hline \multicolumn{2}{|c|}{$f(x)$} & $\boldsymbol{x}_{\mathbf{0}}$ & \multicolumn{5}{|c|}{ COC } \\
\hline \multicolumn{2}{|c|}{} & $\boldsymbol{N M}$ & $\boldsymbol{M 3}$ & $\boldsymbol{P M}$ & $\boldsymbol{M K} 7$ & $\boldsymbol{G M}$ \\
\hline$(a)$ & -1.5 & 2 & 6 & 6 & 7 & 7 \\
\hline$(b)$ & 5 & 2 & 6 & 6 & 7 & 7 \\
\hline$(c)$ & 1 & 2 & 6 & 6 & 7 & 7 \\
\hline
\end{tabular}

\begin{tabular}{|l|l|l|l|l|l|l|}
\hline$(d)$ & 5 & 2 & 6 & 6 & 7 & 7 \\
\hline$(e)$ & 3 & 2 & 6 & 6 & 7 & 7 \\
\hline
\end{tabular}

TABLE II. COMPARISON FF THE ITERATION NUMBER TO APPROXIMATE THE ROOT TO 14 DECIMAL PLACES FOR DIFFERENT ITERATIVE METHOD

\begin{tabular}{|c|c|c|c|c|c|c|}
\hline \multicolumn{2}{|c|}{$f(x)$} & $x_{\mathbf{0}}$ & \multicolumn{5}{|c|}{ N } \\
\hline \multicolumn{2}{|c|}{} & NM & M3 & PM & MK7 & GM \\
\hline$(a)$ & -1.5 & 6 & 3 & 3 & 2 & 2 \\
\hline$(b)$ & 5 & 34 & 14 & 9 & NA & 4 \\
\hline$(c)$ & 1 & 5 & 3 & 2 & 2 & 2 \\
\hline$(d)$ & 5 & 8 & 4 & 3 & 3 & 3 \\
\hline$(e)$ & 3 & 6 & 3 & 2 & NA & 3 \\
\hline
\end{tabular}

TABLE III. COMPARISON OF THE NUMBER OF FUNCTION EVALUATIONS FOR DIFFERENT ITERATIVE METHODS

\begin{tabular}{|c|c|c|c|c|c|c|}
\hline \multicolumn{2}{|c|}{$f(x)$} & $\boldsymbol{x}_{\mathbf{0}}$ & \multicolumn{5}{|c|}{ NOFE } \\
\hline \multicolumn{2}{|c|}{} & NM & $\boldsymbol{M} 3$ & $\boldsymbol{P M}$ & $\boldsymbol{M K} 7$ & $\boldsymbol{G M}$ \\
\hline$(a)$ & -1.5 & 12 & 12 & 12 & 8 & 8 \\
\hline$(b)$ & 5 & 68 & 56 & 36 & NA & 16 \\
\hline$(c)$ & 1 & 10 & 12 & 8 & 8 & 8 \\
\hline$(d)$ & 5 & 16 & 16 & 12 & 12 & 12 \\
\hline$(e)$ & 3 & 12 & 12 & 8 & NA & 12 \\
\hline
\end{tabular}

IV. CONCLUSION

In this paper, we compose the different fourth-order method, Newton's method and the divided difference to obtain new iterative methods with sixth and seventh order convergence. Numerical experiment shows that our methods play at least equal performance compared to other methods with the same convergence order.

\section{ACKNOWLEDGEMENTS}

This research was supported from the National Natural Science Foundation of China (No. 60903143 and 51075421); Natural Science Foundation of Zhejiang Province of China (No. Y1110504); Qianjiang Talent Project of Zhejiang Province of China (No. QJD0902006); Science and Technology Project of Zhejiang Province of China (No. 2012C21035); Young Researchers Foundation of Zhejiang Provincial Top Key Academic Discipline of Mechanical Design and Theory and Zhejiang Sci-Tech University Key Laboratory (ZSTUMD2011B004); Project of Zhejiang Provincial Research Center of Clothing Engineering Technology (2012003). 


\section{REFERENCES}

[1] N.M. Patrikalakis, T. Maekawa, Intersection problems, In: Farin, G., Hoschek, J., Kim, M.S. (Eds.), Handbook of Computer Aided Geomtric Design, Elsevier, 623-649(2002)

[2] M. Barton, B. Juttler, Computing roots of polynomials by quadratic clipping, Computer Aided Geometric Design, 24, 125-141(2007)

[3] L. Liu, Z. Zhang, B. Lin, G. Wang, Fast approach for computing roots of polynomials using cubic clipping, Computer Aided Geo metric Design, 26, 547-559(2009)

[4] J.F. Traub. Iterative Methods for the solution of Equations, Chelsea Publishing, New York (1982)

[5] S.K. Parhi, D.K. Gupta. A six order method for nonlinear equations. Applied Mathematics and Computation, 203, 50-55 (2008)
[6] S. Weerakoon, T.G.I. Fernando. A variant of Newton's method with accelerated third-order convergence. Applied Mathematics Letters, 13, 87-93 (2000)

[7] J. R. Sharma, R. K. Guha. A family of modified Ostrowski methods with accelerated sixth order convergence. Applied Mathematics and Computation, 190, 111-115 (2007)

[8] N.A. Mir, T. Zaman. Some quadrature based three-step iterative methods for non-linear equations. Applied Mathematics and Computation, 193, 366-373 (2007)

[9] A. Cordero, J.L. Hueso. A family of iterative methods with sixth and seventh order convergence for nonlinear equations. Mathematical and Computer Modeling, 52, 1490-1496 (2010)

[10] J. Kou, Y. Li, X. Wang. Some modifications of Newton's method with fifth-order convergence. Journal of Computational and Applied Mathematics, 209, 146-152 (2007)

[11] W.Gautschi. Numerical Analysis: An Introduction, BirKhauser (1997) 